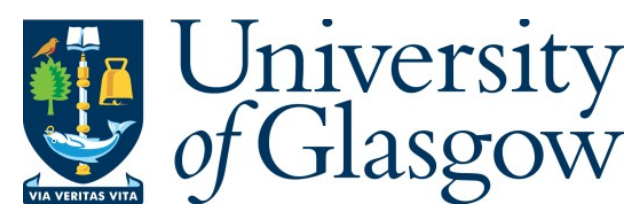

Oberstaller, J., Otto, T. D., Rayner, J. C. and Adams, J. H. (2021) Essential genes of the parasitic apicomplexa. Trends in Parasitology, 37(4), pp. 304-316.

(doi: $10.1016 /$ j.pt.2020.11.007)

This is the Author Accepted Manuscript.

There may be differences between this version and the published version. You are advised to consult the publisher's version if you wish to cite from it.

https://eprints.gla.ac.uk/228993/

Deposited on: 19 January 2021

Enlighten - Research publications by members of the University of Glasgow http://eprints.gla.ac.uk 


\section{Essential genes of the parasitic Apicomplexa}

3 Jenna Oberstaller ${ }^{1}$, Thomas D. Otto 2 , Julian C. Rayner ${ }^{3,4}$, John H. Adams ${ }^{1^{*}}$

$5 \quad{ }^{1}$ Center for Global Health and Infectious Diseases and USF Genomics Program,

6 College of Public Health, University of South Florida, 3720 Spectrum Boulevard, Suite

7 404, Tampa, Florida FL 33612, USA.

$8{ }^{2}$ Centre of Immunobiology, Institute of Infection, Immunity and Inflammation, College of

9 Medical, Veterinary and Life Sciences, University of Glasgow, Glasgow, UK.

10 Malaria Programme, Wellcome Trust Sanger Institute, Genome Campus, Hinxton

11 Cambridgeshire, CB10 1SA, UK.

$12{ }^{4}$ Cambridge Institute for Medical Research, University of Cambridge, Cambridge

13 Biomedical Campus, The Keith Peters Building, Hills Road, Cambridge,

14 Cambridgeshire, CB2 OXY, UK.

15 *Correspondence: ja2@usf.edu (J. H. Adams)

17 Keywords: malaria; Plasmodium falciparum; Plasmodium berghei; Toxoplasma gondii;

18 Apicomplexa; essential genes

\section{Abstract}

21 Genome-scale mutagenesis screens for genes essential for apicomplexan parasite

22 survival have been completed in three species: Plasmodium falciparum, the major

23 human malaria parasite; $P$. berghei, a model rodent malaria parasite, and the more

24 distantly related Toxoplasma gondii, the causative agent of toxoplasmosis. These three

25 species share 2606 single-copy orthologs, 1500 of which have essentiality data in all

26 three screens. In this review, we explore the overlap between these datasets to define

27 the core essential genes of the phylum Apicomplexa. We further discuss the

28 implications of these groundbreaking studies for understanding apicomplexan parasite

29 biology, and we identify promising areas of focus for developing new pan-apicomplexan

30 parasite interventions. 
31 Genome-wide essentiality screens: A game-changer for studying apicomplexan

32 parasite biology

34 Apicomplexan parasites are the causative agents of some of the world's most

35 devastating infectious diseases, including malaria (caused by Plasmodium),

36 toxoplasmosis (Toxoplasma gondii) and numerous other deadly diseases of humans

37 and animals. Though their importance to human health have made these parasites of

38 sustained interest to the research community, many aspects of their biology remain

39 largely mysterious compared to that of more experimentally tractable model organisms.

40 Available tools for genetic manipulation that have worked well in model organisms to

41 functionally characterize genes are typically much more limited in their application to

42 apicomplexan parasites for a number of reasons (summarized in Table 1), leaving

$43 \sim 35 \%$ of malaria-parasite genes and $\sim 50 \%$ of Toxoplasma genes with no functional

44 annotation, even though sequenced genomes in some state of assembly have been

45 available to explore for well over a decade (https://www.veupathdb.org; (1-3)).

46 Overcoming these significant hurdles to explore parasite gene-function is of utmost

47 importance, as a better understanding of parasite biology, and which processes and

48 pathways are more essential for parasite survival, is needed to prioritize targets of more

49 efficacious interventions, particularly in the face of rising parasite resistance to front-line

50 antimalarial drugs.

51

52 Three studies in recent years have vastly accelerated gene-functional characterization

53 by defining the essential genes of three related parasites: Plasmodium falciparum, the

54 most deadly human malaria parasite (4); Plasmodium berghei, a robust rodent malaria

55 parasite that serves as an important model for human malaria (5), and Toxoplasma

56 gondii, a distantly-related parasite responsible for toxoplasmosis whose relative

57 experimental tractability has long led it to be known as the model apicomplexan (6).

58 Similar to other whole-genome essentiality screens in human cells (7-9), essential

59 genes of these parasitic protists are defined by the inability to disrupt protein-coding

60 sequences either by targeted or random mutagenesis methods (see Glossary). The

61 small compact apicomplexan genomes are notable for their extensive signatures of 
62 horizontal gene transfer, especially as a result of the secondary endosymbiotic event

63 giving rise to the plant-like apicoplast organelle, and allowing acquisition of new

64 metabolic pathways (10-12). The complete lack of functional characterization for

65 significant proportions of apicomplexan genes has posed a formidable challenge to 66 unraveling parasite biology; even the remaining genes with annotation deduced by

67 orthology to model organisms largely have sparse functional annotation. Prior to these

68 genome-wide studies, the numbers of genes confirmed to be essential to parasite

69 survival, cumulatively over decades of research and laborious experimentation,

70 numbered only in the 100s (13). With the release of these genome-scale functional

71 studies, researchers now have a clearer picture than ever before about what makes

72 these parasites tick — critical knowledge of parasite biology to possibly weaponize

73 against these diseases via improved interventions (Box 1).

75 In this review, we compare the findings of these three groundbreaking studies to identify

76 shared, essential genes across this diverse phylum. We further note the many shared

77 essential genes that have no identified orthologs in human, making them parasite-

78 specific potential therapeutic targets. Many of these essential genes of unknown

79 function localize to the apicoplast and mitochondria-both of which have been

80 repeatedly demonstrated to be "druggable"-underscoring the roles of these two

81 organelles as rich hubs of essential parasite-specific biology.

82

83 Box 1: What biological properties of parasite genes make them good targets for 84 intervention?

85

86 Drug targets

87 Good parasite drug targets should be essential to parasite survival, as perturbing their

88 function should kill the parasite. Targets should be selective in that they have no or

89 limited similarity to human genes to minimize the chance of harm to the host. Promising

90 drug targets will also ideally operate in druggable pathways outside of those already

91 targeted by frontline antimalarials to engineer multi-faceted therapies against which

92 parasites will have difficulty evolving resistance (14). 


\section{Vaccine targets}

95 Similar considerations for vaccine development in the post-essential-genome era have 96 recently been discussed elsewhere (15) and thus will not be a focus of this review. We

97 will mention only that the purpose of vaccines is to expose the immune system to a 98 threat and prompt it to make inhibitory antibodies or effector immune cells that can be

99 employed in the event of infection. In contrast to the comparatively recent selective 100 pressures drugs have imposed-malaria parasites have co-evolved over millennia to 101 evade the hominid immune system (16), and many potential epitope targets mislead or 102 restrict broadly neutralizing immune inhibition, complicating identification of what is truly 103 a conserved vaccine.

\section{Diverse parasites, one phylum}

Malaria remains a leading global cause of death and severe disease even though rates have fallen dramatically in the past decade (17). Plasmodium falciparum in particular demonstrates an unforeseen intransigence to being eliminated, as evident by its recent

110 rebound; the tide of progress against the disease has plateaued at just over 200 million

111 cases per year, with no significant reduction in the global malaria burden since 2015

112 (17). Emerging drug-resistance in South-East Asia $(18,19)$ once again demonstrates

113 this parasite's ability to evolve resistance to any front-line antimalarial drug, even when

114 given in combination therapies (20). This recurring trend of resistance emphasizes the

115 need for a better understanding of parasite biology to inform use of existing control

116 measures to hold ground against a further malaria resurgence whilst new interventions

117 are developed.

119 Targeted, gene-by-gene functional studies, many prior to availability of full parasite 120 genome sequences and despite extreme limitations in $P$. falciparum in particular, 121 formed the basis for mechanistic understanding of many critical parasite processes 122 (reviewed in (21)). The advent of whole-genome sequencing technologies and 123 publication of the first apicomplexan-parasite genomes enabled larger-scale 
124 characterization than ever before, particularly through comparative genomics, further 125 driving the understanding that these parasites are in many ways in a league of their own 126 biologically, far-removed from model-organisms, governed by different rules shaped by 127 their parasitic lifestyles (for example, (22-26)). However, even with the tremendous 128 advances in understanding the biology of this parasite through targeted gene 129 characterization of important phenotypes (e.g., drug resistance), functional analysis of 130 poorly characterized genes remains a significant challenge, especially for the many 131 hypothetical genes and genes of unknown function. Our newfound ability to 132 characterize targets based on their essentiality amongst diverse apicomplexan species

133 will help prioritize targets and pathways that are most sensitive to functional disruption.

134 In addition, mutant-parasite libraries can help define drug mechanisms of action by 135 chemogenomic profiling of mutants $(27,28)$ and identifying underlying transcriptional 136 changes associated with altered drug sensitivity (29).

138 Plasmodium berghei, the most tractable of the rodent models for malaria for 139 experimental genetics is $\sim 15$ - 35 million years diverged from $P$. falciparum (16, 30, 31). 140 The genetic tool set developed by PlasmoGEM for studying gene function is more 141 developed than that for $P$. falciparum $(5,32,33)$. The proportion of essential genes is 142 surprisingly similar in both species, even though essentiality by in vivo infection for $P$. 143 berghei would be presumably more stringent than the in vitro ideal culture conditions for $144 P$. falciparum. The overlap of orthologs also reflects the general level of homology 145 between these species and is consistent with the accepted value of the rodent malaria 146 model, as demonstrated by discoveries in malaria-parasite core biological functions 147 originating from studies in $P$. berghei, then subsequently replicated with much more 148 effort in $P$. falciparum (e.g., ApiAP2G $(34,35)$ ).

150 Toxoplasmosis is a widespread zoonotic disease of world-wide social and economic 151 importance, an opportunistic pathogen which can infect and destroy any nucleated cell, 152 with dire consequences primarily for the immuno-compromised and unborn (36). Widely 153 viewed as the "model" apicomplexan parasite because of the sophistication of available 154 genetic tools and ease of genetic manipulation, Toxoplasma gondii often serves as an 
155 experimental system for malaria parasites (37), even though $T$. gondii diverged $~ 500-$ 156900 million years from Plasmodium spp. (38). While this considerable divergence in

157 time is at a similar scale as ancestral chordates to modern humans (39), T. gondii

158 shares core biological properties with Plasmodium spp.; both are obligate-intracellular

159 parasites that undergo asexual replication within human cells and have common host-

160 cell invasion, replication and egress processes (Table 1). Divergence does place limits

161 on what can be extrapolated from this model to Plasmodium, especially in dissecting

162 more recent evolutionary adaptations of malaria parasites. Nonetheless, the conserved

163 aspects of biology and underlying genetics among apicomplexans in these

164 evolutionarily distant organisms allow powerful insight into what it means to be an

165 essential apicomplexan gene. In our three-way species comparison of essentiality data,

166 we identify core, essential apicomplexan genes with the acknowledgement that many of

167 these essential genes have likely taken on new, possibly distinct functions in the

168 different species.

\section{Comparing essentiality data across organisms/screens}

172 The principles behind determining gene essentiality and dispensability from all three

173 screens are similar. Genes that tolerate disruption of their protein-coding sequence

174 (CDS) are dispensable whereas parasites die when the CDS of essential genes are

175 disrupted, meaning parasites with mutations in these genes are not recovered. All the

176 apicomplexan parasite screens involve large-scale mutagenesis to generate single-

177 gene mutations per parasite, each with unique sequence signatures making them

178 identifiable in a pool. Pooled mutants were then subjected to phenotypic screening via

179 competitive growth assays. Analysis of all three assays utilized some form of high-

180 throughput, Next-Gen sequencing to assess depletion in reads for mutants in the pool

181 as a proxy for growth, with more reads indicating a more successful mutant (and

182 therefore dispensable gene), and fewer reads/the absence of reads indicating sicker

183 mutants and essential genes. 
185 By definition, all genes in any orthogroup are descended from a single ancestral gene 186 having the same sequence and function. As gene duplications and losses happen 187 frequently over the course of evolution with complex outcomes for function that cannot 188 be readily inferred, we limited our essentiality comparisons to orthologous genes with a 189 single representative in each of the three parasites (single-copy orthologs) which had 190 essentiality data in all three screens. Of the 2606 single-copy orthologs shared between 191 these three parasites, 1500 had essentiality data in all three screens and were used for 192 our comparative analyses (Supplementary Table S1).

194 Essentiality across genome-scale assays: what does it mean to be an essential 195 apicomplexan gene?

197 Despite these commonalities, methodological differences in how the essentiality data 198 were collected and interpreted for each apicomplexan screen necessitate defining a 199 common language for assigning essentiality vs. dispensability for the purposes of 200 comparison. All three assays assigned some sliding measure of confidence to assign 201 essentiality category, with some genes falling clearly into the "essential" or 202 "dispensable" categories for the tested conditions, and many more in the grey-area of 203 uncertainty as we consider methodology/assay-specific caveats for the interpretation of 204 each dataset. We sought to define the most high-confidence essential and dispensable 205 genes from each assay for a conservative comparison of essentiality and dispensability 206 across these parasites.

208 In the case of the $P$. falciparum screen, inherent properties of individual genes 209 independent of essentiality can affect the likelihood of recovering a piggyBac

210 transposon insertion in that gene, such as gene length and TTAA density. The piggyBac 211 transposon preferentially inserts at TTAA sites, and while these sites are for the most

212 part quite abundant and evenly distributed across the $P$. falciparum genome (averaging 213 one site every $~ 80 \mathrm{bp}$; (4)), we took caution in designating genes with low TTAA density 214 ( $<7$ TTAA sites $/ \mathrm{kb}$ ) as essential based on the absence of recovered insertions alone.

215 Similarly, there may be decreased chances of recovering insertions from very short 
216 genes $(<500 \mathrm{bp})$ independent of essentiality. We therefore designated the $<13 \%$ of

217 genes falling in to either of these categories as "tentative" and did not consider them for

218 comparison.

219

220 Methods of the $P$. berghei and $T$. gondii screens were similar, utilizing targeted, non-

221 homologous recombination techniques to generate a library of mutants. The $P$. berghei

222 screen is the most limited in terms of number of genes for which essentiality was

223 characterized (at 2578 genes, $>50 \%$ of the genome), through the use of targeted, non-

224 homologous recombination techniques to excise the full coding-region of a gene.

225 Mutants exhibited a range of growth phenotypes, and mutants characterized as having

226 a less-conclusive "slow" growth phenotype as compared to wild-type parasites were not

227 considered in our comparisons. The authors were additionally careful to note any

228 "essential" designations resulting from mutants generated via vectors having particularly

229 short homology-arms, which is known to decrease recombination efficiency, as less-

230 conclusive $(5,40)$. We filtered these genes from our comparisons. In T. gondii a

231 CRISPR-based screen was feasible due to the parasite's high rates of non-homologous

232 end-joining, allowing for higher-throughput targeted mutagenesis methods. Individual

233 in-depth follow-up phenotype studies of individual mutant parasites supported the

234 overall conclusions of the whole-genome profiling.

236 Direct comparisons using simple essentiality-score cutoffs to assign gene

237 essentiality/dispensability across assays suggested misleadingly low correlation in

238 essentiality-classifications across these parasites than would be expected by overall

239 orthology. We therefore used a ranking-method to binarily classify genes as essential or

240 dispensable, ranking orthologous genes by sliding-scale essentiality score in each

241 screen (Mutagenesis Index Score, or MIS, for P. falciparum; Relative Growth Rate,

242 or RGR, for P. berghei, and Phenotype Score for T. gondii), then further reducing the

243 dataset to genes ranking in the top-and bottom-quantiles for all three screens, ensuring

244 a conservative comparison of only the most-confidently classified essential and

245 dispensable genes. The final gene set for the three-species comparison comprised 816

246 genes with a high degree of correlation in essentiality between screens (ranging from 
$24774.5 \%$ to $81.6 \%$ ), more consistent with overall orthology (Figure 1A-C; Supplementary 248 Table S2).

Specialized apicomplexan organelles are rich with essential, uncharacterized parasite biology

253 Broadly conserved categories of genes within each apicomplexan species tend to be 254 essential in all three screens (Figure 2, Key Figure; Supplementary Table S3). Not

255 surprisingly, many of the conserved apicomplexan essential processes have also been

256 found to be essential in very distantly-related eukaryotes such as yeast, mice, and

257 humans, particularly translation-associated functions and cellular components such as

258 the ribosome (7-9, 41-44). Similarly, core metabolic pathways for RNA metabolism

259 appear highly conserved in all eukaryotic organisms and are unsurprisingly essential

260 across apicomplexans, while motor-activity and signaling pathways are primarily

261 dispensable. Essential vs dispensable gene-sets within each organism displayed the 262 generally expected characteristics for these gene categories in agreement with other

263 essentiality screens; essential genes compared to non-essential genes tend to be more

264 deeply conserved, have lower occurrence of polymorphisms, and have lower 265 redundancy (no paralogs that might compensate for loss-of-function) (4-6, 45). The

266 broad consensus across the growing number of essentiality-screens serves as

267 confirmation of methodology for defining the apicomplexan essential-gene repertoire. 268

269 Beyond these expected essential gene sets of broadly conserved genes are 132 270 apicomplexan-essential genes that have little or no functional annotation because they

271 do not have enough similarity to studied genes in model organisms. Several of these

272 essential unknowns have evidence for being targeted to the apicoplast (Supplementary

273 Table S3). At least $10 \%$ of apicomplexan-essential genes are comprised of

274 mitochondria and apicoplast-targeted genes.

275

276 The complex evolutionary origins of the apicomplexan mitochondrion and apicoplast

277 (which arose from endosymbiosis of an alpha-proteobacterium and red-algal 
278 endosymbiont, respectively $(24,46)$ and thus have little overlap with human proteins)

279 have long made these organelles promising drug targets. Both of these endosymbionts,

280 over the course of hundreds of millions of years of evolution, became intimately

281 integrated into parasite biology, housing some of the most highly essential pathways to

282 parasite survival (Figure 2; Supplementary Table S3). Given the unusual origins of

283 these pathways and adaptations the ancestral parasite had to make to interface with

284 and regulate these new organelles_associated genes are rich with parasite-specific,

285 essential yet unexplored functions, and processes mapping to these organelles are

286 indeed overrepresented among shared-essential genes.

287

288 Essential apicomplexan-specialized mitochondrial processes

289

290 Many basic mitochondrial functions are conserved and essential across eukaryotes,

291 including the generation of ATP, iron-sulfur cluster synthesis, or redox regulation (47);

292 indeed, we find key genes underlying these conserved processes among shared-

293 essential genes in our comparison (Figure 2; Supplementary Table S2-S3). Though it is

294 generally accepted that all eukaryotic mitochondria arose from the same endosymbiotic

295 event, there are striking functional differences in mitochondrial functions and pathways

296 across eukaryotes, with apicomplexan lineages having among the most-reduced

297 mitochondrial genomes studied (comprising only three protein-coding genes, and highly

298 fragmented bits of ribosomal RNA genes) (47-49). The majority of mitochondrial

299 proteins are thus nuclear-encoded, necessitating these parasites develop complex

300 systems of signaling, protein-import and export to regulate this organelle (50).

301

302 There is strong evidence to suggest translation does happen within the parasite

303 mitochondria, and that somehow the fragmented mitochondrially-encoded rRNAs get

304 assembled into a functional ribosome $(51,52)$. Factors regulating these complex

305 processes were largely unknown. Plastid and mitochondrial proteins are known to be

306 heavily post-transcriptionally regulated in plants, with a largely-expanded family of

307 organelle-targeted, nuclear-encoded RNA-binding proteins, the pentatricopeptide-repeat

308 (PPR) proteins, responsible for most of this regulation (53). Plasmodium has two 
309 annotated PPR proteins, one of which is targeted to the apicoplast (PfPPR1;

310 PF3D7_1406400/PBANKA_1035800) and the other does not have a clear targeting

311 sequence for either the apicoplast or the mitochondria (PfPPR2;

312 PF3D7_1233300/PBANKA_1448000/TGGT1_243530). Both are predicted to be highly

313 essential in Plasmodium, and though PfPPR1 does not have a direct ortholog in $T$.

314 gondii, $T$. gondii also appears to have a single apicoplast-targeted PPR protein

315 (TGGT1_244050) that is essential for parasite growth (54). Using models based on

316 plant PPR-proteins and the related algal octatricopeptide-repeat (OPR) proteins (which

317 do not have annotated representatives in the Apicomplexa), Hillebrand and colleagues

318 recently discovered a novel family of related proteins they termed heptatricopeptide-

319 repeat (HPR) proteins that they then confirmed to localize to mitochondria and have

320 specific RNA-binding capability (55).

322 Subsequent searches for proteins containing HPR domains across the tree of life 323 indicated representatives across green algae, alveolates (including most members of 324 the Apicomplexa, their photosynthetic relatives the chromerids, and the more distantly325 related dinoflagellates), as well as a small, more divergent handful in human,

326 suggesting the family was an ancient acquisition of the eukaryotic ancestor; however

327 the family has only expanded in green algae and chromalveolates. Phyletic distribution

328 suggested lineage-specific expansion in lineages with more highly fragmented

329 mitochondrial rRNA genes, such as Plasmodium and T. gondii, while representatives

330 from the sister-alveolate ciliate clade, known to have a much more straightforward

331 mitochondrial rRNA-organization, were much fewer. No HPR-domains were detected in

332 basal-branching apicomplexan Cryptosporidium, which has only the remnants of a 333 mitochondrion.

335 These findings taken together strongly suggest the apicomplexan HPR proteins are 336 important regulatory components of the organellar (and particularly, mitochondrial)

337 translation-machinery, and they provide another example of a plant-like innovation in

338 these parasites that could possibly be exploited to human advantage. The

339 approximately 20 HPR proteins in Plasmodium and the 25 in T. gondii for the most part 
340 did not meet the essentiality criteria to be included in our comparisons. However, 341 several representatives were phenotypically characterized in each screen and strongly 342 tend towards being essential. Further, four HPR proteins are shared-essential across all 343 three screens with no human orthologs, while only one is shared-dispensable 344 (Supplementary Table S4). One of four HPR proteins predicted to localize to the 345 apicoplast at their initial discovery has since been verified (PF3D7_0930100), and it too 346 was scored as highly essential in $P$. falciparum. The $P$. berghei ortholog

347 (PBANKA_0830800) was not included in the essentiality screen, and there is no $T$. 348 gondii ortholog. It is also interesting to note that the majority of these HPR proteins were 349 annotated as "hypothetical" at the publication of the essentiality screens, further 350 speaking to apicomplexan endosymbiotic-organelle biology as a frontier of essential 351 functions awaiting characterization.

353 Essential apicoplast processes

355 The apicoplast has long been accepted as essential for parasite survival, though the 356 reasons why were somewhat mysterious (12). Why would a plastid organelle be 357 essential for the survival of a non-photosynthetic, obligate-intracellular organism? 358 Research over the last decade has illuminated that apicoplast-minus mutants can be 359 completely chemically rescued by supplementation with a single intermediate molecule 360 acting within the isoprenoid biosynthesis pathway, isopentenyl pyrophosphate (IPP)

361 (56). This discovery has since enabled development of small-scale phenotypic

362 screening assays of chemically-generated mutant libraries for apicoplast-minus

363 mutants, which were then rescued with IPP for sequencing to determine genes

364 essential for apicoplast biogenesis (57). Apicoplast-minus screening has also been 365 employed to examine drug mechanism of action $(58,59)$.

367 Indispensable Conserved Apicomplexan Proteins (ICAPs): Functional studies. 
369 While the essentiality classification provides one of the most useful basic metrics about 370 these many apicomplexan-specific uncharacterized genes, further functional annotation 371 will require assays to identify their essential functions.

373 Further such investigations to demonstrate the existence of apicomplexan-specific essential processes and to identify promising, parasite-specific drug targets were initiated in the T. gondii CRISPR-mutagenesis screen (6). A set of $\sim 200$ genes, termed "indispensable conserved apicomplexan proteins (ICAPs)", were identified to be apicomplexan-specific and essential, though the entire list of these genes was not explicitly delineated. Of the 17 ICAPs Sidik et al. specified, 14 have single-copy orthologs across $P$. falciparum and $P$. berghei, and half of them have essentiality predictions in all three species. Sidik et al. further molecularly characterized these 17 ICAPs, noting their localization and confirming essentiality by independently generated

382 mutants (Supplementary Table S5). Interestingly, the majority of these proteins were 383 localized to discrete compartments.

385 Temporal and spatial characterization of unknown proteins can be quite informative in 386 apicomplexans, especially for the invasive motile stages, since their discrete organelles 387 and other internal compartments often are associated with specialized functions (e.g., 388 micronemes sequester invasion ligands (60)). For example, based on the initial $T$. 389 gondii phenotype, secondary phenotyping with conditional knock-outs in $P$. falciparum 390 using regulatable gene modifications provided verification of cross-species essentiality 391 of a claudin-like apicomplexan microneme protein (CLAMP)(6). Additional consensus-

392 based $P$. falciparum essentiality data and experimental studies of ICAPS should 393 broaden support for essentiality of potentially druggable targets ripe for investigation.

394 Similarly, half of those ICAPs that could be phenotypically categorized in the P. berghei 395 screen were essential or slow-growers, including the CLAMP ortholog (5).

397 Endosymbiont-derived organelles house a large proportion of apicomplexan-specific 398 functions. Large-scale essentiality screens validate that these functions are highly 399 enriched in shared, essential genes, many of which do not have human orthologs. Many 
400 of these shared, essential genes were annotated as "hypothetical" at publication of the 401 large-scale essentiality screens, and even still at the time of this review. Apicomplexan402 specific essential processes have been revealed several times to have emerged from 403 co-opting distantly related protein-domains picked up along the way from their bacterial 404 and algal endosymbionts (such as the HPR-family of organelle-targeted RNA-binding 405 proteins, or the ApiAP2 family of master transcriptional regulatory proteins; $(55,61$, 406 62) ). In light of these observations, it stands to reason that further experimental 407 assessment of parasite-specific, shared-essential hypothetical genes will reveal even 408 more organelle-specific functions and parasite biology.

Towards a nuanced interpretation of target-essentiality and specificity in rational, targetbased anti-parasitic drug-development

413 A promising drug target does not necessarily have to be free of human orthologs;

414 indeed there are several examples of promising drugs that were engineered to 415 specifically target parasite orthologs of widely conserved essential proteins with no 416 reported ill-effects on the orthologous human protein (for example, (63)). As there are 417 no definitive guidelines regarding the acceptable level of conservation between host and 418 parasite proteins before off-target effects become a concern in drug-development, 419 genes with no identified orthologs in the human host are a conservative place to start in 420 the search for viable targets. Endosymbiont-derived organelles are hotbeds of essential, 421 apicomplexan-specific functions, many performed by genes without human orthologs 422 (Supplementary Table S4, S6).

424 Several effective antimalarial drugs of the past have been found to target mitochondrial 425 or apicoplast pathways; enthusiasm for these targets has waxed, waned, and waxed 426 again as we assemble a more and more detailed puzzle of parasite biology 427 necessitating an evolving, nuanced interpretation of just what makes a good target.

428 Drugs known to target the housekeeping functions of mitochondrial or apicoplast 429 pathways generally result in a "delayed death" phenotype, meaning parasites are able 430 to undergo one round asexual replication before death, which made these drugs of 
431 lower priority as current drug-development guidelines prioritize fast-acting drugs that 432 decrease parasite chances of developing resistance. Recent studies have identified 433 promising apicoplast or mitochondrial targets that swiftly kill the parasite within a single

434 cycle, however, which has revived enthusiasm for these organelles as potential sources 435 of fast-killing targets (64).

437 Resistance against the mitochondrial electron transport chain (ETC) enzyme 438 cytochrome b-targeted drug Atovaquone evolved alarmingly rapidly, particularly 439 disappointing given its initial efficacy and highly safe toxicology profile. Atovaquone's 440 failure dulled excitement for other mitochondria-related drug-targets $(65,66)$. That 441 resistance, however, was not transmissible due to biological peculiarities of the 442 parasite-functions of the mitochondria are more essential during mosquito-stage 443 development, and the very characteristics that made resistance rapidly arise in blood444 stage therapies made it an effective transmission-blocking possibility $(65,67)$.

445 Moreover, recent studies have found atovaquone to be effective in combinatorial 446 therapy with another drug targeting the same component of the mitochondrial ETC in 447 another active site (68), against which resistance did not evolve experimentally. 448 Atovaquone-synergistic effects were not seen with another compound targeting 449 cytochrome $\mathrm{b}(68)$. These findings suggest not only that mitochondrial pathways retain 450 value for antimalarial intervention - but that combinatorial therapies employing drugs 451 acting synergistically against the same target may also prove to be worth-while. Given 452 that vast compound-libraries screened for antimalarial activity and then interrogated for 453 their mechanism of action have largely converged on the same handful of target454 proteins, despite great chemodiversity in these libraries (69), further evaluation of these 455 drugs in concert is warranted.

457 Mitochondrial-targeted proteins that are encoded in the single nuclear genome will have 458 different properties to consider as potential drug-targets than mitochondrial genome459 encoded proteins, and have other strengths-the single nuclear genome (as opposed to $460 \sim 22$ mitochondrial genomes per Plasmodium parasite) mean there is less likelihood that 461 low-frequency population heterogeneity can allow a beneficial mutation to rise to 
462 fixation, as was the case with atovaquone (66). The three essentiality screens targeted

463 only nuclear-encoded genes, identifying many shared-essential, mitochondrial-targeted

464 candidate genes (Figure 2; Supplementary Table S2, S3 \& S4).

465 Notably, the most successful past and present front-line antimalarials (chloroquine and 466 artemisinin, respectively) are themselves excellent examples of exploitation of parasite467 specific biology/endosymbiont-derived organelles, with both drugs relying in part on 468 vesicular trafficking of host-cell hemoglobin to the digestive vacuole for parasite-killing 469 activity - a process ultimately dependent upon apicoplast-enabled post-translational 470 modifications to Rab-family vesicular trafficking proteins (70-75).

472 Though concepts are emerging of what it means to be an "essential" gene with the 473 availability of more and more screens across the tree of life (and even synthetic life;

474 (76) ) - it is clear that essentiality is context-dependent and cannot truly be binarily 475 assigned (77), particularly in parasitic organisms where evolution by genome-reduction 476 is the rule, and every gene is likely essential in an environment-dependent context 477 (such as host, developmental stage, environmental stressors such as host fever or 478 antimalarials). In addition to the conditional nature of essentiality, there are other 479 properties of genes and genomes that necessitate a nuanced interpretation to these 480 screens. Essentiality is not a fixed property of genes themselves; genes function as 481 components in the larger service of metabolic pathways and biological processes, and 482 evidence suggests these components can be and often are substituted(77). Indeed 483 there are only a few handful of genes themselves that are shared across all eukaryotes 484 and can be traced back to the Last Eukaryotic Common Ancestor (LECA) (78), mostly 485 related to translation; however essentiality of core processes necessary to sustain life 486 remains conserved. Thus essentiality is a plastic characteristic of genes, and all 487 essential genes are not created equal—defects in some essential genes can be 488 compensated for more easily than defects in others (79). 
492 We focused this review on shared characteristics across the three organisms, but

493 contrasts in gene-essentiality between them can also be quite informative and reflect

494 the vastly different niches these parasites have evolved to fill; for instance, the TCA

495 cycle is essential in T. gondii, but dispensable during the Plasmodium blood stages,

496 which instead relies on glycolytic metabolism to generate its energy (Supplementary

497 Table S7-S12). Plasmodium-specific contrasts suggest the relative importance of

498 apicoplast and mitochondrial pathways in these two parasites, with apicoplast fatty acid

499 biosynthesis and a selection of mitochondrial genes essential in P. falciparum, yet

500 dispensable in $P$. berghei (Supplementary Table S3 \& S9). These apparent differences

501 in essentiality of certain organellar pathways within Plasmodium spp. may possibly

502 reflect differences in requirements for in vitro vs in vivo infection. Biological relevance of

503 these and other indicated differences in essential processes require further

504 investigation.

505

506 The essential/dispensable screens provide critical initial phenotype-data for most

507 parasite genes under ideal growth conditions. However, the power of the resources

508 generated in these screens-particularly the expansive libraries of uniquely identifiable,

509 single-gene mutant parasites - extends far beyond answering the relatively broad

510 question of parasite gene-essentiality during select developmental stages (see

511 Outstanding Questions). These initial essentiality phenotypes will serve as the

512 backbone for developing and interpreting screens for any number of phenotypes of

513 interest, potentially allowing high-throughput functional characterization for thousands of

514 genes at a time. These screens may enable the research-community to iteratively

515 ascribe function to the vast majority of apicomplexan parasite genes over time,

516 unlimited by a priori functional knowledge.

518 We are now in the post essential-genome era of apicomplexan parasite research.

519 Forward-genetic screening utilizing these expansive mutant-parasite resources makes

520 an accelerated pace for functional biological breakthroughs possible, or perhaps likely.

521 Such insights have the potential to drive discovery of new druggable targets, or to

522 enable thoughtful application of existing interventions as synergistic co-therapies. 


\section{Glossary}

526 Endosymbiont-derived organelles: the organelles arising from the endosymbiosis of 527 an algal or bacterial cell in the apicomplexan parasite ancestor-the apicoplast and the 528 mitochondria, respectively.

529

530 Mutagenesis Index Score (MIS): The sliding-scale score developed by (4) used as the 531 primary metric for assessing comparative essentiality of $P$. falciparum genes.

533 Orthologs: Genes in different species that originated by vertical descent from a single

534 gene of the last common ancestor. All copies of genes descended from the same 535 ortholog make up an ortholog group.

537 Paralogs: Genes within the same genome that belong to the same ortholog group.

538 Paralogs result from a gene duplication-event in evolutionary history somewhere

539 downstream from the last eukaryotic common ancestor.

540

541 Phenotype Score: The sliding-scale score developed by (6) used as the primary metric 542 for assessing comparative essentiality of $T$. gondii genes.

543

544 Random mutagenesis: Mutagenesis method usually utilizing a transposon that inserts 545 preferentially into a single, random occurrence of its given recognition sequence per 546 genome (in the case of the piggyBac transposon used for the $P$. falciparum essentiality

547 screen, TTAA). Random mutagenesis is particularly useful for exploring highly repetitive 548 genomes where large-scale utilization of targeted mutagenesis, which requires long 549 stretches of homologous sequence for target-specificity and recombination efficiency, is 550 impractical (such as the AT-rich $P$. falciparum genome).

552 Relative Growth Rate (RGR): The sliding-scale score developed by (5) used as the 553 primary metric for assessing comparative essentiality of $P$. berghei genes. 
555 Shared-essential genes: Single-copy orthologs confidently scored as essential in all

556 three essentiality screens.

558 Single-copy orthologs: Also known as one-to-one orthologs. Single-copy ortholog

559 groups have exactly one gene copy in each species being compared. As all genes in

560 any orthogroup are descended from a single ancestral gene, with the same sequence

561 and function, while duplications and loss happen comparatively frequently—single-copy

562 orthologs are a less-complex subset of the wider dataset that still contains all the

563 information of the complete dataset. Single-copy orthologs are the most useful for

564 evolutionary comparisons, as we do not have to make any inferences about the

565 ancestral state (or the order in which gene duplication or loss occurred based on

566 presence or absence of paralogs).

567

568 Targeted mutagenesis: Mutagenesis method utilizing plasmid vectors designed to edit

569 a specific gene, usually by homologous recombination (e.g., CRISPR). Requires

570 comparatively long homology regions with the target-gene for efficiency ( $1 \mathrm{~kb}$ on each

571 side of the target-region), and as such has high target-specificity. Both the T. gondii and

572 P. berghei essentiality screens utilized a form of targeted mutagenesis.

573

574

\section{Acknowledgements}

576 We thank our colleagues Dr. Chengqi Wang, Dr. Rays Jiang, Swamy Rakesh Adapa,

577 members of the Adams lab, and the USF Genomics Program Omics Hub for critical

578 discussion that improved the quality of this work. We thank the Kissinger lab at the

579 University of Georgia for extended methodological discussions and tips. Computational

580 analyses were performed using resources provided or maintained by the USF

581 Department of Research Computing. This work was supported by the National Institutes

582 of Health grants F32-Al112271 (awarded to J.O.), R01 Al094973 and R01 Al117017

583 (awarded to J.H.A.) 


\section{References}

585 1. M. J. Gardner et al., Genome sequence of the human malaria parasite Plasmodium falciparum. Nature 419, 498-511 (2002). gondii genome. Nucleic acids research 31, 234-236 (2003).

3. U. Böhme, T. Otto, M. Sanders, C. Newbold, M. Berriman, Progression of the canonical reference malaria parasite genome from 2002?2019 [version 2; peer review: 3

593

4. M. Zhang et al., Uncovering the essential genes of the human malaria parasite

594

595

596

597

598

599

600

601

602

603

604

605

606

607

608

609

610

611

612

613

614

615

616

617

618

619

620

621

622

5. E. Bushell et al., Functional Profiling of a Plasmodium Genome Reveals an Abundance of Essential Genes. Cell 170, 260-272.e268 (2017).

6. S. M. Sidik et al., A Genome-wide CRISPR Screen in Toxoplasma Identifies Essential Apicomplexan Genes. Cell 166, 1423-1435.e1412 (2016).

7. T. Wang et al., Identification and characterization of essential genes in the human genome. Science 350, 1096-1101 (2015).

8. V. A. Blomen et al., Gene essentiality and synthetic lethality in haploid human cells. Science 350, 1092-1096 (2015).

9. T. Hart et al., High-Resolution CRISPR Screens Reveal Fitness Genes and GenotypeSpecific Cancer Liabilities. Cell 163, 1515-1526 (2015).

10. J. Huang, N. Mullapudi, T. Sicheritz-Ponten, J. C. Kissinger, A first glimpse into the pattern and scale of gene transfer in Apicomplexa. Int J Parasitol 34, 265-274 (2004).

11. L. Lim, G. I. McFadden, The evolution, metabolism and functions of the apicoplast. Philosophical transactions of the Royal Society of London. Series B, Biological sciences 365, 749-763 (2010).

12. B. Striepen, The apicoplast: a red alga in human parasites. Essays in biochemistry 51, 111-125 (2011).

13. J. White, P. K. Rathod, Indispensable malaria genes. Science 360, 490-491 (2018).

14. M. J. Chaparro et al., Efforts Aimed To Reduce Attrition in Antimalarial Drug Discovery: A Systematic Evaluation of the Current Antimalarial Targets Portfolio. ACS Infectious Diseases 4, 568-576 (2018).

15. C. Proietti, D. L. Doolan, The case for a rational genome-based vaccine against malaria. Front Microbiol 5, 741 (2014).

16. M. A. Pacheco, M. Cranfield, K. Cameron, A. A. Escalante, Malarial parasite diversity in chimpanzees: the value of comparative approaches to ascertain the evolution of Plasmodium falciparum antigens. Malaria Journal 12, 328 (2013).

17. WHO, World Malaria Report. World Health Organization, (2018).

18. F. Ariey et al., A molecular marker of artemisinin-resistant Plasmodium falciparum malaria. Nature 505, 50-55 (2014).

623

624

19. O. Miotto et al., Multiple populations of artemisinin-resistant Plasmodium falciparum in

625 Cambodia. Nature genetics 45, 648-655 (2013). resistance among Cambodian Plasmodium falciparum. Nat Commun 9, 1769 (2018). 
627 21. A. E. Sexton, C. Doerig, D. J. Creek, T. G. Carvalho, Post-Genomic Approaches to Understanding Malaria Parasite Biology: Linking Genes to Biological Functions. ACS Infectious Diseases 5, 1269-1278 (2019).

630

22. J. D. DeBarry, J. C. Kissinger, Jumbled genomes: missing Apicomplexan synteny. Mol Biol

631

632

633 Evol 28, 2855-2871 (2011).

634

635

636

637

638

639

23. J. D. DeBarry, J. C. Kissinger, A survey of innovation through duplication in the reduced
genomes of twelve parasites. PLoS One 9, e99213-e99213 (2014).

24. J. Janouškovec, A. Horák, M. Oborník, J. Lukeš, P. J. Keeling, A common red algal origin of the apicomplexan, dinoflagellate, and heterokont plastids. Proceedings of the National Academy of Sciences 107, 10949 (2010).

25. Y. H. Woo et al., Chromerid genomes reveal the evolutionary path from photosynthetic algae to obligate intracellular parasites. Elife 4, e06974 (2015).

640

26. S. Balaji, M. M. Babu, L. M. Iyer, L. Aravind, Discovery of the principal specific transcription factors of Apicomplexa and their implication for the evolution of the AP2integrase DNA binding domains. Nucleic Acids Res 33, 3994-4006 (2005).

27. A. Pradhan et al., Chemogenomic profiling of Plasmodium falciparum as a tool to aid antimalarial drug discovery. Sci Rep 5, 15930 (2015).

28. W. C. Van Voorhis et al., Open Source Drug Discovery with the Malaria Box Compound

Collection for Neglected Diseases and Beyond. PLoS Pathog 12, e1005763 (2016).

29. J. Gibbons et al., Altered expression of K13 disrupts DNA replication and repair in Plasmodium falciparum. BMC Genomics 19, 849 (2018).

30. S. Kumar, G. Stecher, M. Suleski, S. B. Hedges, TimeTree: A Resource for Timelines, Timetrees, and Divergence Times. Mol Biol Evol 34, 1812-1819 (2017).

31. U. Böhme et al., Complete avian malaria parasite genomes reveal features associated with lineage-specific evolution in birds and mammals. Genome Research, (2018).

32. T. Sanderson, J. C. Rayner, PhenoPlasm: a database of disruption phenotypes for malaria parasite genes. Wellcome Open Res 2, 45 (2017).

33. F. Schwach et al., PlasmoGEM, a database supporting a community resource for largescale experimental genetics in malaria parasites. Nucleic Acids Res 43, D1176-1182 (2015).

34. A. Sinha et al., A cascade of DNA-binding proteins for sexual commitment and development in Plasmodium. Nature 507, 253-257 (2014).

660

661

662

663

664

35. B. F. Kafsack et al., A transcriptional switch underlies commitment to sexual

666

667

668 development in malaria parasites. Nature 507, 248-252 (2014).

36. V. B. Carruthers, Host cell invasion by the opportunistic pathogen Toxoplasma gondii. Acta Tropica 81, 111-122 (2002).

37. K. Kim, L. M. Weiss, Toxoplasma gondii: the model apicomplexan. International Journal for Parasitology 34, 423-432 (2004).

38. E. J. Douzery, E. A. Snell, E. Bapteste, F. Delsuc, H. Philippe, The timing of eukaryotic evolution: does a relaxed molecular clock reconcile proteins and fossils? Proc Natl Acad Sci U S A 101, 15386-15391 (2004).

669 tunicates. BMC Biol 16, 39 (2018). 
670 40. A. R. Gomes et al., A genome-scale vector resource enables high-throughput reverse genetic screening in a malaria parasite. Cell Host Microbe 17, 404-413 (2015).

672

41. H. Chen et al., New insights on human essential genes based on integrated analysis and
the construction of the HEGIAP web-based platform. Briefings in Bioinformatics 21,

674 1397-1410 (2019).

675

42. M. Kabir, A. Barradas, G. T. Tzotzos, K. E. Hentges, A. J. Doig, Properties of genes

676 essential for mouse development. PLoS One 12, e0178273 (2017).

677

678

43. G. Giaever et al., Functional profiling of the Saccharomyces cerevisiae genome. Nature

679 418, 387-391 (2002).

680

44. Jacqueline K. White et al., Genome-wide Generation and Systematic Phenotyping of

681 Knockout Mice Reveals New Roles for Many Genes. Cell 154, 452-464 (2013).

682

45. W.-H. Chen, K. Trachana, M. J. Lercher, P. Bork, Younger Genes Are Less Likely to Be

683

684 Essential than Older Genes, and Duplicates Are Less Likely to Be Essential than Singletons of the Same Age. Molecular Biology and Evolution 29, 1703-1706 (2012).

685

46. A. J. Roger, S. A. Muñoz-Gómez, R. Kamikawa, The Origin and Diversification of

686

687 Mitochondria. Current Biology 27, R1177-R1192 (2017).

47. A. B. Vaidya, M. W. Mather, Mitochondrial Evolution and Functions in Malaria Parasites. Annual Review of Microbiology 63, 249-267 (2009).

688

689

690

48. L. Sheiner, A. B. Vaidya, G. I. McFadden, The metabolic roles of the endosymbiotic organelles of Toxoplasma and Plasmodium spp. Current Opinion in Microbiology 16,

691

692

693

694

695 452-458 (2013).

49. H. J. Santos, T. Makiuchi, T. Nozaki, Reinventing an Organelle: The Reduced Mitochondrion in Parasitic Protists. Trends in Parasitology, (2018).

50. N. Mallo, J. Fellows, C. Johnson, L. Sheiner, Protein Import into the Endosymbiotic Organelles of Apicomplexan Parasites. Genes (Basel) 9, 412 (2018).

696

697

698

699

700

701

702

703

704

705

706

707

708

709

710

711

712

713

51. P. Pino et al., Mitochondrial translation in absence of local tRNA aminoacylation and methionyl tRNA Met formylation in Apicomplexa. Mol Microbiol 76, 706-718 (2010).

52. A. Sharma, A. Sharma, Plasmodium falciparum mitochondria import tRNAs along with an active phenylalanyl-tRNA synthetase. Biochem J 465, 459-469 (2015).

53. J. Colcombet et al., Systematic study of subcellular localization of Arabidopsis PPR proteins confirms a massive targeting to organelles. RNA Biol 10, 1557-1575 (2013).

54. J. L. Hicks et al., An essential pentatricopeptide repeat protein in the apicomplexan remnant chloroplast. Cellular Microbiology 21, e13108 (2019).

55. A. Hillebrand et al., Identification of clustered organellar short (cos) RNAs and of a conserved family of organellar RNA-binding proteins, the heptatricopeptide repeat proteins, in the malaria parasite. Nucleic Acids Res, (2018).

56. E. Yeh, J. L. DeRisi, Chemical Rescue of Malaria Parasites Lacking an Apicoplast Defines Organelle Function in Blood-Stage Plasmodium falciparum. PLoS Biology 9, e1001138 (2011).

57. Y. Tang et al., A mutagenesis screen for essential plastid biogenesis genes in human malaria parasites. PLOS Biology 17, e3000136 (2019).

58. W. Wu et al., A chemical rescue screen identifies a Plasmodium falciparum apicoplast inhibitor targeting MEP isoprenoid precursor biosynthesis. Antimicrobial agents and chemotherapy 59, 356-364 (2015). 
714

715

716

717

718

719

720

721

722

723

724

725

726

727

728

729

730

731

732

733

734

735

736

737

738

739

740

741

742

743

744

745

746

747

748

749

750

751

752

753

754

755

756

757
59. K. Amberg-Johnson et al., Small molecule inhibition of apicomplexan FtsH1 disrupts plastid biogenesis in human pathogens. elife 6, e29865 (2017).

60. M.-J. Gubbels, M. T. Duraisingh, Evolution of apicomplexan secretory organelles. International journal for parasitology 42, 1071-1081 (2012).

61. J. Oberstaller, Y. Pumpalova, A. Schieler, M. Llinas, J. C. Kissinger, The Cryptosporidium parvum ApiAP2 gene family: insights into the evolution of apicomplexan AP2 regulatory systems. Nucleic Acids Res 42, 8271-8284 (2014).

62. K. Modrzynska et al., A Knockout Screen of ApiAP2 Genes Reveals Networks of Interacting Transcriptional Regulators Controlling the Plasmodium Life Cycle. Cell host \& microbe 21, 11-22 (2017).

63. J. E. Gisselberg, Z. Herrera, L. M. Orchard, M. Llinas, E. Yeh, Specific Inhibition of the Bifunctional Farnesyl/Geranylgeranyl Diphosphate Synthase in Malaria Parasites via a New Small-Molecule Binding Site. Cell chemical biology 25, 185-193.e185 (2018).

64. M. J. Boucher, E. Yeh, Disruption of Apicoplast Biogenesis by Chemical Stabilization of an Imported Protein Evades the Delayed-Death Phenotype in Malaria Parasites. mSphere 4, e00710-00718 (2019).

65. C. D. Goodman, H. D. Buchanan, G. I. McFadden, Is the Mitochondrion a Good Malaria Drug Target? Trends Parasitol 33, 185-193 (2017).

66. S. Siegel et al., Mitochondrial heteroplasmy is responsible for Atovaquone drug resistance in Plasmodium falciparum. bioRxiv, 232033 (2017).

67. C. D. Goodman et al., Parasites resistant to the antimalarial atovaquone fail to transmit by mosquitoes. Science 352, 349 (2016).

68. A. M. Stickles et al., Atovaquone and ELQ-300 Combination Therapy as a Novel Dual-Site Cytochrome Inhibition Strategy for Malaria. Antimicrobial agents and chemotherapy 60, 4853 (2016).

69. J. White, P. K. Rathod, Indispensable malaria genes. Science 360, 490 (2018).

70. J. Birnbaum et al., A Kelch13-defined endocytosis pathway mediates artemisinin resistance in malaria parasites. Science 367, 51 (2020).

71. N. F. Gnädig et al., Insights into the intracellular localization, protein associations and artemisinin resistance properties of Plasmodium falciparum K13. PLoS Pathog 16, e1008482 (2020).

72. K. F. Suazo, C. Schaber, C. C. Palsuledesai, A. R. Odom John, M. D. Distefano, Global proteomic analysis of prenylated proteins in Plasmodium falciparum using an alkynemodified isoprenoid analogue. Scientific Reports 6, 38615 (2016).

73. L. Imlay, A. R. Odom, Isoprenoid Metabolism in Apicomplexan Parasites. Current Clinical Microbiology Reports 1, 37-50 (2014).

74. D. J. Sullivan, Jr., I. Y. Gluzman, D. G. Russell, D. E. Goldberg, On the molecular mechanism of chloroquine's antimalarial action. Proceedings of the National Academy of Sciences of the United States of America 93, 11865-11870 (1996).

75. K. Kennedy et al., Delayed death in the malaria parasite Plasmodium falciparum is caused by disruption of prenylation-dependent intracellular trafficking. PLOS Biology 17, e3000376 (2019).

76. M. Juhas, L. Eberl, G. M. Church, Essential genes as antimicrobial targets and cornerstones of synthetic biology. Trends in Biotechnology 30, 601-607 (2012). 
758 77. G. Rancati, J. Moffat, A. Typas, N. Pavelka, Emerging and evolving concepts in gene $759 \quad$ essentiality. Nature Reviews Genetics 19, 34 (2017).

760 78. E. V. Koonin, Comparative genomics, minimal gene-sets and the last universal common 761 ancestor. Nat Rev Microbiol 1, 127-136 (2003).

762 79. G. Liu et al., Gene Essentiality Is a Quantitative Property Linked to Cellular Evolvability. 763 Cell 163, 1388-1399 (2015).

764 80. D. M. Emms, S. Kelly, OrthoFinder: phylogenetic orthology inference for comparative 765 genomics. Genome Biology 20, 238 (2019).

766 


\section{Table 1. General comparisons across apicomplexans.}

769

770

\begin{tabular}{|c|c|c|c|}
\hline Characteristic & P. falciparum(3D7) & P. berghei (ANKA) & Toxoplasma gondii (GT1) \\
\hline Primary host & mosquito & mosquito & cat \\
\hline Secondary host & humans & mice & most mammals \\
\hline infected cell type & red blood cell & red blood cell & epithelial cell \\
\hline $\begin{array}{l}\text { Genome size/protein- } \\
\text { coding gene number }\end{array}$ & $23.33 \mathrm{MB} / 5460$ genes & $18.78 \mathrm{MB} / 5067$ genes & $63.95 \mathrm{MB} / 8460$ genes \\
\hline $\begin{array}{c}\text { Advantages as } \\
\text { experimental system }\end{array}$ & the deadliest human malaria parasite & $\begin{array}{c}\text { relative genetic } \\
\text { tractability; in vivo model } \\
\text { for human malaria } \\
\text { infection }\end{array}$ & $\begin{array}{l}\text { most genetic tractability; } \\
\text { the "model" apicomplexan, } \\
\text { and important pathogen in } \\
\text { its own right }\end{array}$ \\
\hline $\begin{array}{l}\text { Disadvantages as } \\
\text { experimental system }\end{array}$ & $\begin{array}{c}\text { difficult to genetically manipulate; very } \\
\text { low transfection efficiency; high- } \\
\text { throughput targeted mutagenesis } \\
\text { unlikely due to repetitive genome } \\
\text { composition }\end{array}$ & $\begin{array}{c}\text { not the human malaria } \\
\text { parasite; no in vitro } \\
\text { system }\end{array}$ & $\begin{array}{l}\text { far-removed from malaria } \\
\text { parasites; limits to how } \\
\text { much can be extrapolated } \\
\text { to Plasmodium }\end{array}$ \\
\hline Mutagenesis method & $\begin{array}{l}\text { random transposon-mediated } \\
\text { insertional mutagenesis } \\
\text { (Zhang et al. 2018) }\end{array}$ & $\begin{array}{l}\text { targeted mutagenesis } \\
\text { (Bushell et al. 2017) }\end{array}$ & $\begin{array}{l}\text { targeted mutagenesis } \\
\text { (Sidik et al. 2016) }\end{array}$ \\
\hline $\begin{array}{l}\% \text { of genes assigned } \\
\text { essentiality score }\end{array}$ & $87 \%$ & $50 \%$ & $\sim 95 \%$ \\
\hline Essentiality scoring metric & Mutagenesis Index Score (MIS) & $\begin{array}{l}\text { Relative Growth Rate } \\
\text { (RGR) }\end{array}$ & Phenotype score \\
\hline studied stage & in vitro blood stage & in vivo blood stage & in vitro lytic cycle \\
\hline \multicolumn{4}{|l|}{ Total shared genes* } \\
\hline$P f$ & -- & 4631 & 2928 \\
\hline$P b$ & 4581 & -- & 2861 \\
\hline$T g$ & 3044 & 3028 & -- \\
\hline
\end{tabular}

$771{ }^{*}$ Numbers of shared genes between species vary by direction of comparison (reflecting

772 different numbers of within-species paralogs in ortholog groups).

773

774

775 
776 Figure 1. Genes included in essentiality comparisons for this review with added

777 evolutionary context. A. Species ortholog-overlaps and genes retained after each

778 filtering step. Total gene-numbers for each organism are indicated in parentheses. Of

7792606 single-copy orthologs shared between all three parasites, 1500 had phenotype

780 data from all three essentiality screens. Our comparison-set comprised 816 genes

781 confidently scored in all three screens. Single-copy orthologroups between each pair of

782 species are also reported ( $P f / P b, 4508 ; P f / T g, 2668 ; P b / T g, 2653)$. B-C. Phylogenetic

783 relatedness of $P$. falciparum, $P$. berghei, and $T$. gondii and their hosts, respectively.

784 Amphioxus, a basal-branching marine chordate, has a similar scale of evolutionary

785 distance from humans/rats as $T$. gondii has from Plasmodium (included for evolutionary

786 reference). Evolutionary timescales are median estimates of divergence time based on

787 several publications as calculated by timetree.org (30).

788

789 Figure 2, KEY FIGURE. Comparison and contrast between select essential

790 pathways across parasites as determined by enrichment analyses of each

791 essentiality category. Cladograms on the right indicate each essentiality category,

792 where orange indicates essentiality and black indicates dispensability for the given

793 species. The $y$-axis (-log2 $p$-value) describes the absolute significance of a term's

794 enrichment, and the $x$-axis (Z-score) describes the significance of each term compared

795 to every other significant term in that essentiality category. The upper left quadrant of

796 each category indicates highest significance (lowest Z-score and highest -log2 p-value

797 (weighted Fisher/elim hybrid test)). Only significant terms ( $p$-value $<=0.05$ ) are plotted.

798 Coordinates are approximate (points are staggered randomly for ease of viewing). Font-

799 size and point-size indicate the number of significant genes in the essentiality category

800 mapping to that GO-term. Expected highly conserved categories such as translation-

801 associated terms are highly essential across all three parasites, as are terms related to

802 endosymbiont-derived organelles (highlighted in red). BP: biological process. CC:

803 cellular component. MF: molecular function.

804

805

806 


\section{Supplementary Files}

808 File 1:

809

810 Table S1. All single-copy orthogroups between $P$. falciparum $(P f), P$. berghei $(P b)$, and

811 T. gondii $(\mathrm{Tg})$, and essentiality-phenotype information from each of the three screens

812 where available. Orthogroups and single-copy orthologs were defined using the

813 OrthoFinder algorithm with default parameters $(80)$.

814

815 Table S2. All Pf-Pb-Tg single-copy orthologs which have phenotype scores above

816 confidence-threshold in all essentiality screens $(n=816)$. Genes were ranked by

817 essentiality phenotype-score in each respective screen (by Mutagenesis Index-Score

818 (MIS) for Pf; Relative Growth Rate (RGR) for Pb, and mean phenotype-score for $\mathrm{Tg}$ ). All

819 but the top-scoring (most essential) 60\% and 30\% bottom-scoring (least essential)

820 genes for each screen were filtered out. Genes confidently scored in each of the

821 screens were assigned binary scores of 1 (indicating "essential") or 2 (indicating

822 "dispensable") for further functional analysis.

823

824 Table S3. Each essentiality profile was tested for GO-term functional enrichment 825 against a background of all 816 genes in the analysis. Significant GO terms (weighted 826 Fisher/elim-hybrid test p.value $<=0.05$ ) are reported.

827

828 Table S4. 132 genes out of the 816-gene comparison-set are (1) essential in all three 829 phenotype-screens and (2) have no human orthologs (as determined by orthoFinder). 830

831 Table S5. Single-copy orthologs of previously determined Indispensable Conserved 832 Apicomplexan Proteins (ICAPs) and available phenotype information from each screen. 833 These previously-unannotated genes were investigated via targeted disruption in $T$. 834 gondii to confirm essentiality and assess localization. The phenotype scoring for the 835 majority of these genes across all three assays did not meet our stringent requirements 836 for comparison, but many are likely potential genes of interest, particularly those for 837 which essentiality is likely in $P f$ and $P b$. 
839 Table S6. The full orthoFinder-determined ortholog lists of the 132 shared-essential

840 genes with no human orthologs from the 816-gene comparison set across select

841 apicomplexans and outgroup chromerids.

842

843 File 2:

844

845 Table S7. All single-copy orthologs shared between $P$. falciparum and P. berghei. 846

847 Table S8. All $P f-P b$ single-copy orthologs which have phenotype scores above 848 confidence-threshold in both essentiality screens. Genes were ranked by essentiality 849 phenotype-score in each respective screen (by Mutagenesis Index-Score (MIS) for Pf, 850 and Relative Growth Rate (RGR) for $P b$ ). Top-scoring (most essential) $60 \%$ and $30 \%$ 851 bottom-scoring (least essential) genes for each screen were assigned binary scores of 1 852 (indicating "essential") or 2 (indicating "dispensible") for further functional analysis.

854 Table S9. Each essentiality category was tested for GO-term functional enrichment 855 against a background of all 1407 genes in the analysis. Significant GO terms (weighted856 Fisher/elim-hybrid test p.value $<=0.05$ ) are reported.

858 Table S10. All single-copy orthologs shared between $P$. falciparum and T. gondii. 859

860 Table S11. All Pf-Tg single-copy orthologs which have phenotype scores above 861 confidence-threshold in both essentiality screens. Genes were ranked by essentiality 862 phenotype-score in each respective screen (by Mutagenesis Index-Score (MIS) for Pf, 863 and Mean Phenotype Score for Tg). Top-scoring (most essential) 60\% and 30\% bottom864 scoring (least essential) genes for each screen were assigned binary scores of 1 865 (indicating "essential") or 2 (indicating "dispensable") for further functional analysis. 
867 Table S12. Each essentiality category was tested for GO-term functional enrichment 868 against a background of all 1856 genes in the analysis. Significant GO terms (weighted869 Fisher/elim-hybrid test p.value $<=0.05$ ) are reported. 